\title{
e-Phaïstos
}

e-Phaïstos

Revue d'histoire des techniques / Journal of the history

of technology

I-2 | 2012

Les sources de l'histoire des techniques

\section{Automobilismes. Visite des clichés de l'immobilité}

Automobilisms. Beyond the "clichés" of immobility

\section{Mathieu Flonneau}

\section{OpenEdition}

Journals

\section{Édition électronique}

URL : http://journals.openedition.org/ephaistos/421

DOI : 10.4000/ephaistos.421

ISSN : 2552-0741

\section{Éditeur}

IHMC - Institut d'histoire moderne et contemporaine (UMR 8066)

\section{Édition imprimée}

Date de publication : 1 décembre 2012

Pagination : 71-75

ISSN : 2262-7340

Référence électronique

Mathieu Flonneau, "Automobilismes. Visite des clichés de l'immobilité », e-Phaïstos [En ligne],

I-2 | 2012, mis en ligne le 12 février 2016, consulté le 23 avril 2019. URL : http://

journals.openedition.org/ephaistos/421 ; DOI : 10.4000/ephaistos.421 


\title{
Automobilismes Visite des clichés de l'immobilité
}

\author{
Mathieu Flonneau \\ Maître de conférences \\ IRICE - UMR 8138 \\ P2M \\ Université Paris I Panthéon-Sorbonne
}

«L'automobile ne peut plus accomplir aucun rêve de mouvement inconnu : c'est un objet désormais immobile »

Roland BARTHES, « La voiture, projection de l'ego », Réalités, nº 213, 1963.

\section{Introduction}

En dehors de sa consommation, l'objet technique automobile n'existe pas. In vitro, l'auto n'est qu'une abstraction : elle n'a de réalité que sortie des chaînes et en situation. L'artefact mobile est nécessairement consommé, usé, voire abusé. C'est précisément cette perspective que Thomas Mailaender explore d'une façon très originale dans ses Cathedral Cars récemment rassemblées en volume grand format et que nous proposons ici de commenter ${ }^{1}$.

Penser : ce ne sont que des voitures. L'erreur serait là. Même si, après tout, bien sûr... Mais croire que l'auto, en certaines circonstances, n'est pas beaucoup plus que l'auto, condamne à manquer la vie et la chaleur fortement présentes dans chacune des illustrations glacées de ce bel ouvrage pourtant dénué de toutes présences ou figures humaines.

L'écriture à froid peut-elle restituer la vie de ces éléments mouvants de décor à destination des pays chauds ? Essayons au moins d'en relever le pari.

\section{Petite iconosophie d'automobiles improbables}

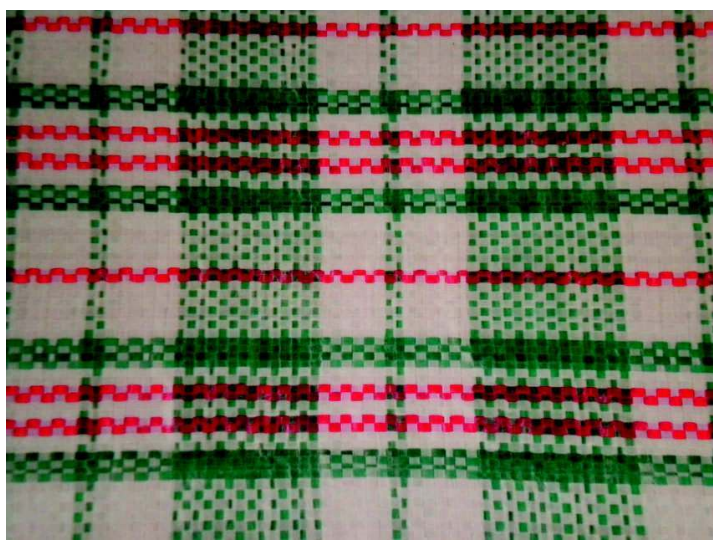

Fig. 1 : "Tartan Tati", gage d'anoblissement de pauvres mais dignes automobiles

Le « tartan Tati » est éloquent et leur donne à chacune des lettres de noblesse. Présentes sous forme de sac sur presque toutes les galeries ou bien visibles dans les coffres surchargés, les toiles plastifiées très 
solides bleues et rouges, ou vertes et rouges c'est selon, sont comme un signe de reconnaissance de ces automobiles de seconde zone. Plus élégantes en tous cas que les simples bâches unies bleues ou vertes aussi utilisées, elles égayent ce tiers-monde automobile dont la puissance suggestive est considérable.

Elles participent toutes d'une même série artistique, inattendue et suggestive, celle des Cathedral Cars. Baptisées ainsi, ces « voitures cathédrales » ont été saisies par Thomas Mailaender, docker occasionnel, infiltré - légalement ! - sur les terminus du port de Marseille, porte méditerranéenne de l'Europe vers l'Afrique.

L'artiste les a immortalisées en 2005 et a repris pour les dénommer l'appellation que les professionnels des installations portuaires leur ont spontanément donnée. Mieux que personne, par son effort compulsif, il en a compris le sens qu'il donne avec bonheur à partager. À notre tour de creuser le cliché et d'essayer de dépasser la lecture automobile de ces saynètes. Ce qui fait sens en l'occurrence dépasse de beaucoup le dérisoire des quatre roues, de l'esthétique incertaine des carrosseries bosselées et des émanations sonores et fumigènes du moteur qui va avec (dans le meilleur des cas, celui d'origine est bien fatigué...).

Ce qui fait sens dans ces voitures est au-delà d'elles : c'est la dimension politique et géopolitique de ces faits sociaux totaux que les automobiles, de quelques espèces qu'elles ressortent, incarnent sans rivales depuis le début du siècle dernier. Belle longévité pour un objet au plus haut point culturel que d'aucuns affectent de tenir pour insignifiant ou régressif.

Il s'agit de voitures cathédrales donc : voitures aux chargements si audacieux qu'ils font naturellement songer aux élancements gothiques vers le divin des transepts et travées, vitraux et voûtes des bâtisseurs du Moyen Âge. Voitures toutes en hauteur, même si elles tendent à toucher la chaussée, édifices fragiles et éphémères certes, mais aussi voitures inspirées pour lesquelles la foi sert de tendeur ultime. Foi en une vie meilleure, croyance dans un ailleurs si loin et pourtant si proche.
Voitures cathédrales en effet. Belle appellation, qui parle aussi aux connaisseurs lettrés des mondes de l'automobilisme. Nous y reviendrons.

Ces véhicules ouvrent une fenêtre sur un tout autre automobilisme, il faut bien le dire, que celui plus classique que fréquentent plutôt les spécialistes de la chose roulante, historiens compris ! Le dédain amusé - assurément moins que du mépris en tout état de cause - est inévitablement présent en général lorsqu'un " vrai » amateur d'automobiles est confronté à ce type de tacot improbable quoique très ordinaire.

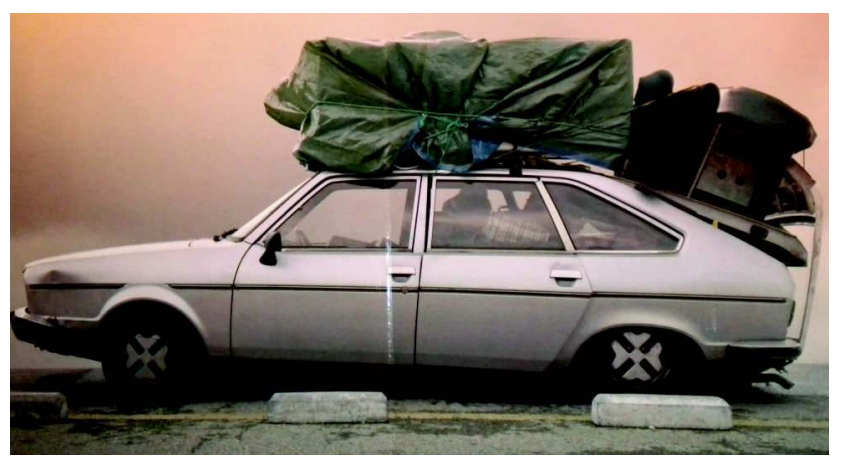

Fig. 2 : Renault 20 surchargée, "Tête haute" !

Fondamentalement, qui peut s'extasier devant ces outils de mobilités romanichelles : une Renault Express si chargée au cul que son pot touche terre ? une R2O " tête haute » à son coffre défendant ? une Peugeot 204 hors d'âge au pare-choc défoncé et au bric-à-brac vacillant?

Ces carrosseries harnachées parlent pourtant à notre imaginaire. Tous, nous en avons vu. Nos départs ensoleillés et embouteillés en vacances croisaient ces transhumances d'un autre genre parfois annuelles souvent plus rares encore. On n'en voit plus de nos jours ou de moins en moins à l'évidence... Cette rareté nouvelle s'explique par le fait que la normalisation technique des parcs automobiles est passée par là. Toutefois elles existent toujours. Sur une autoroute ou une nationale à petite vitesse, au détour d'une aire de repos, sur la bande d'arrêt d'urgence parfois, capot levé ou cric déployé, nous les reconnaissons de loin. Hors périodes 
de congés, dans « le civil », à Paris comme à Marseille, dans les banlieues des grandes villes, au cours de l'année, elles restent identifiables : elles font parfois " échouées de trottoirs » - comme chez les amateurs on parle avec déférence des « sorties de grange ». Il leur arrive aussi de faire la fierté de leur propriétaire sur les parkings de grands ensembles ${ }^{2}$.

Les ports, lieux intermédiaires et louches par excellence, endroits de stations, et de passages, qui ouvrent vers des voyages qu'un esprit romanesque a vite fait de parer d'énigmatiques vertus, offrent des aires de stationnement et de parkings où l'on en voit encore. Il y a là une petite porte (une portière !) ouverte aux trafics et aux soupçons, aux peurs irrationnelles - quoique pas toutes - également ${ }^{3}$.

Voitures de peu, précaires, rebutantes, risibles à l'occasion, authentiques « bagnoles » - au sens gaulois du terme... -, les voitures cathédrales ont une forme d'aura que cette photographie ingénue et presque drôle, mais pas innocente car le sourire se crispe rapidement, restitue parfaitement. Toutes les petites organisations si révélatrice de l'ensemble d'une existence se retrouvent derrière : vies minuscules en apparence, y compris celles des familles nombreuses, mais que le retour réussi au bled peut transformer en celles de rois du pétrole !

$\mathrm{Au}$ tableau des fétiches ${ }^{4}$ automobilisés, elles ont un droit de cité plein et entier au même titre que les taxis comparables en puissances imaginaires ${ }^{5}$, ou bien à l'autre extrémité du genre, les clichés de Michael Furman, portraitiste attitré de la collection d'élite Ralph Lauren ${ }^{6}$. Après tout, ces prises de vue cliniques au fond neutre, choisi délibérément par l'artiste pour décharger le trop plein parasitaire de la charge pathétique, ne dérogeraient pas dans les pages studio des plus beaux magazines automobiles.

Il reste qu'échappées de leur cadre de papier, pour le dire tout net, nous serions curieux d'en voir rouler certaines !... D'ailleurs, en toute rigueur, parmi ces sculptures potentiellement cinétiques, aux chargements de rêve ou de cauchemar (!) pour un ingénieur des Mines averti du dépassement dangereux du PTAC (poids total autorisé en charge), d'aucunes ne rouleront jamais plus que quelques mètres, sans leur moteur quelque fois inutilisable depuis longtemps -, simplement sur leur élan, poussées à bord des ferrys et re-débarquées au point d'arrivée. En effet, elles ne servent pour certaines d'entre elles que de mules. Le prix de la traversée rend rentable le fait d'utiliser ces conteneurs mobiles pour ramener au pays des matériaux, des objets et des denrées rares. Cette abondance ponctuelle est en réalité le signe révélateur des grands déséquilibres Nord-Sud et des nombreuses pénuries qui affectent l'autre monde. Ces formes bizarres révèlent surtout en creux une économie informelle prédominante.

Le sourire se fige effectivement si l'on lit ces autos à l'aune d'une histoire des choses banales, de la matérialité, bref une histoire de l'avoir qui est en l'occurrence une contre-histoire (comme existent des contre-allées) du sous-développement. En cela, ces approches croisent celles développées par l'historiographie de l'immigration déjà bien renseignée et établie par ailleurs. Ce sont effectivement les mondes parallèles des déplacements humains que les voitures cathédrales racontent : elles en disent long sur les pénuries, les marchés noirs, les secondes, troisièmes et quatrièmes mains, les petits trafics, les libertés défaillantes, etc.

Le monde moderne de l'automobile, celui qui implique l'électronique et l'informatique n'est pas le leur. Il y a d'ailleurs un motif à inquiétude à ce sujet : ce qui pouvait se réparer à même la rue, avec des outils de fortune et ce qui ne nécessitait aucune formation particulière n'est plus de mise. Les deuxièmes, troisièmes et quatrièmes vies de ces dinosaures techniques ne peuvent plus être prolongées. À court terme désormais, faute de prolongation de plus en plus impossible de la vie d'objets techniques de plus en plus surannés, l'Afrique va devoir s'inventer un autre avenir automobile. Une chose est certaine : la Françafrique automobile, celle initiée par les croisières Citroën, celle des pick up Pigeot et du Paris-Dakar7 est, comme les autres, révolue. Les plus optimistes y verront peut-être l'occasion de ruptures inédites, pourquoi pas technologiques, et de nouveaux départs. 

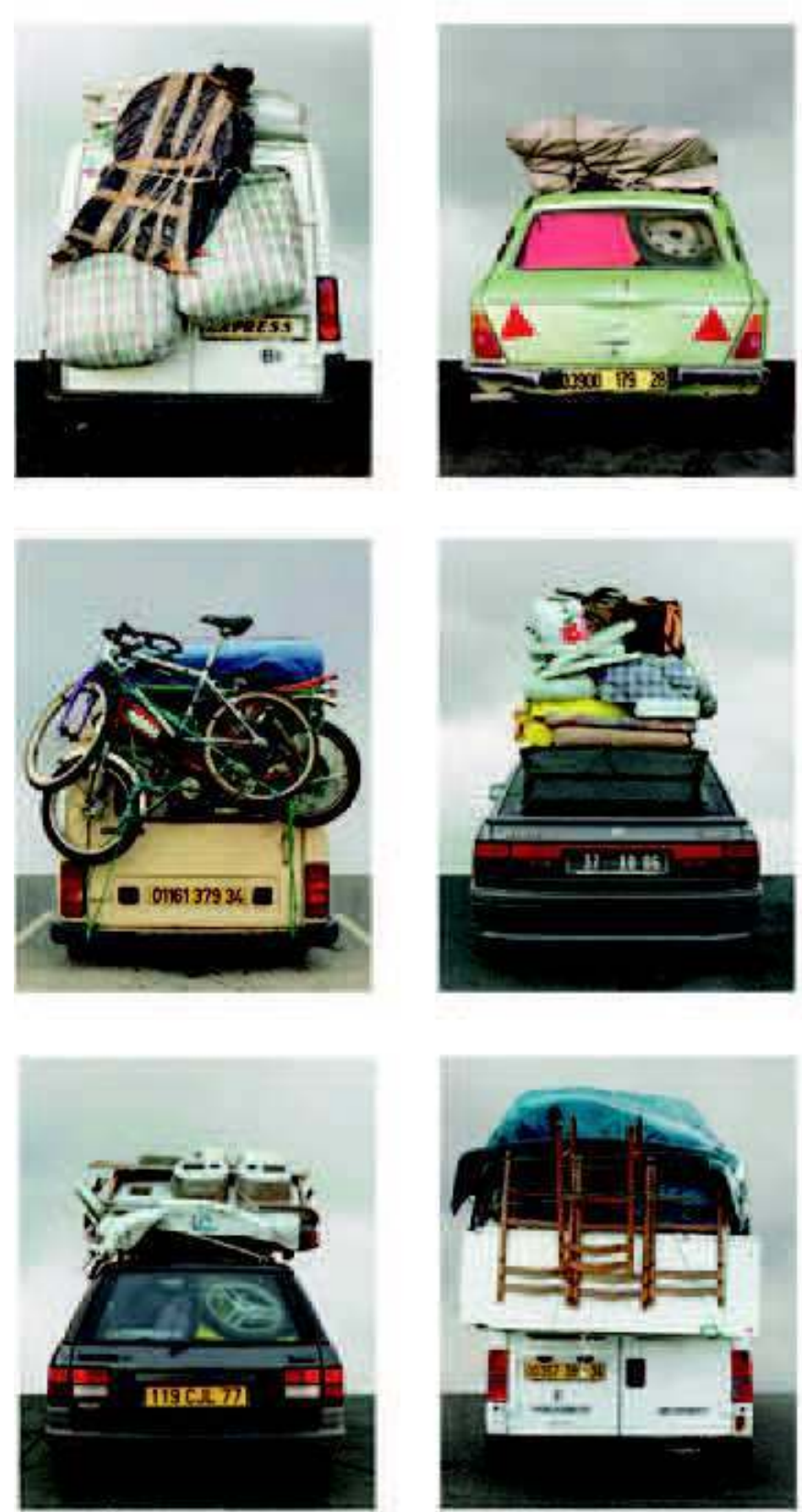

Fig. 3 : Diverses Cathedral Cars : le voyage commence...

\section{Histoires de mobilités : dis-moi comment tu charges ta voiture...}

La toile " tartanée Tati » par son aspect rêche et assez bon marché quoique solide dit aussi d'autres choses : le monde suggéré et entrevu ici n'est pas sans rugosités ni aspérités. Visiter le cliché des cathédrales modernes de la mobilité peut donc se faire du côté automobile de façon presque primaire, mais ces usages baroques de l'objet automobile en disent plus.

Le trop plein ici étalé est en vérité un trop peu ; dans l'économie des voyages contemporains, qu'ils soient automobiles, ferrés ou aériens, seul l'occidental ou le très aisé occidentalisé peut se permettre de voyager léger et sans bagage. C'est un autre monde de chaleur humaine qui transpire dans ces pages, celui de métiers manuels dont on peut supposer l'exercice du côté des propriétaires.

Elles disent la pluralité des mondes automobiles, des modes de voyage, des modes de transport. Elles nous rappellent que dans le kaléidoscope annuel des milliards de mobilités mondiales, les plus nombreuses passent inaperçues. Ces voitures disent la mondialisation à l'œuvre en marge d'un monde occidental aseptisé et blasé vis-à-vis de l'objet automobile et qui oublie que sous d'autres latitudes, il reste désirable, car indispensable aux survies individuelles, familiales et à celle des petites communautés ${ }^{8}$.

On l'oublierait presque : l'automobile permet le retrait vis-à-vis de mobilités assignées et contraintes et loin d'un dévoiement quelconque de l'essence auto-mobile, ces autos en servent vraiment l'idéal.

Finissons-en avec un motif connu : ces voitures ne sont pas que des autos. Elles sont investies de mythologies diverses, comme les vraies cathédrales. Très connue sur ce registre est la comparaison esquissée en 1957 par Roland Barthes, et qui mérite enfin d'être rappelée. Le célèbre sémiologue avait eu l'idée de comparer l'automobile à une mythologie contemporaine. En l'occurrence, il s'agissait d'une DS Citroën.

« Je crois que l'automobile est l'équivalent assez exact des grandes cathédrales gothiques : je veux dire une grande création d'époque, conçue passionnément par 
des artistes inconnus, consommée dans son image, sinon dans son usage, par un peuple entier qui s'approprie en elle un objet parfaitement magique ${ }^{9}$.

Croire que les autos ne parlent pas est d'une naïveté confondante. Ces objets roulants sont aussi des objets parlants de toute évidence. Il n'est bien sûr pas question de la voix féminine d'un si moderne GPS déroulant maussadement mais impérativement un itinéraire, il est question des récits qui y sont afférents et qui pour leur part laissent place à l'inconnu et à la rencontre. Thomas Mailaender l'a compris. Mieux que quiconque peut-être et en se laissant impressionner, à son tour il nous impressionne. En voiture donc ! même si trouver une place confortable à bord ne sera pas forcément très aisé. Il existe cependant une hospitalité automobile que l'on devrait cultiver.

${ }^{1}$ Thomas MAILAENDER, Cathedral Cars, RVB Books/The Fun Archives Publications, 2011. Nous remercions vivement l'auteur et l'éditeur de nous avoir autorisés à publier les photos qui illustrent cet article.

${ }^{2} \mathrm{Cf}$. la thèse de sociologie de Dominique Lefrançois, Le parking dans le grand ensemble, Institut d'urbanisme de Paris, Prix Jean Panhard Automobile et Société, CCFA/ACF, 2008.

${ }^{3}$ Mike DAVIS, Petite histoire de la voiture piégée, éd. Zones, 2007.

${ }^{4}$ Musée Tinguely de Bâle, Voiture fétiche. Je conduis, donc je suis, 2011.

${ }^{5}$ Une référence récente pour le Sud, l'Egypte en l'occurrence : Khaled AL KHAMISSI, Taxi, Acte Sud, (2007), 2009.

${ }^{6}$ L'art de l'automobile. Chefs-d'œuvre de la collection Ralph Lauren, Les Arts Décoratifs, 2011.

${ }^{7} \mathrm{Cf}$. notre production radiophonique pour France-Culture : L'inévitable mirage. Les erreurs de navigation du Paris-Dakar, La Nouvelle Fabrique de l'Histoire, 2011.

${ }^{8}$ Il n'est pas surprenant de voir plusieurs voitures-cathédrales figurer dans l'exposition « J'ai deux amours » (novembre 2011-juin2012), présentée à la Cité nationale de l'immigration.

${ }_{9}^{9}$ Roland BARTHES, Mythologies, Paris, Seuil, 1957. 